\title{
PREVALÊNCIA DE SINTOMAS DEPRESSIVOS E/OU ANSIOSOS EM PESSOAS COM HIPERTENSÃO ARTERIAL SISTÊMICA E I OU DIABETES MELLITUS
}

| Gabriela Neves Paula de Souza'; Rauni Jandé Roama Alves²; Luís Paulo Souza e Souza ${ }^{3}$; Alcindo José Rosa ${ }^{4}$ |

\section{RESUMO}

CONTEXTO: A Hipertensão Arterial Sistêmica (HAS) e o Diabetes Mellitus (DM) têm sido apontados como condições que favorecem o desenvolvimento de transtornos emocionais.

OBJETIVO: Investigar a prevalência de sintomas depressivos e/ou ansiosos (SDA) em pessoas com HAS e/ou DM cadastradas em uma Estratégia Saúde da Família do centro-oeste brasileiro.

MÉTODOS: Trata-se de um estudo transversal e quantitativo. Foram investigados 106 adultos com HAS/DM, aos quais foram aplicados dois instrumentos: (1)Mini International Neuropsychiatric Interview, para avaliação dos SDA; (2) questionário sobre dados socioeconômicos, antropométricos, clínicos e adesão medicamentosa, para caracterização da amostra.

RESULTADOS: Os principais achados indicaram a prevalência de SDA de 27,4\% (IC95\% 19,1-36,8; n = 29) na amostra total. Além disso, tais sintomas foram mais prevalentes nos seguintes estratos dessa população: mulheres (35,4\%; IC95\% 23,7 - 48,6; $\mathrm{n}=22$ ); pessoas com idade mais avançada $(28,5 \%$; IC95\% 14,6 - 46,3; $\mathrm{n}=10)$; pessoas sem companheiros $(36,3 \%$; IC95\% 20,4 - 54,8; $\mathrm{n}=12)$; pessoas que estudaram de zero a oito anos $(27,8 \%$; IC95\% $18,3-39,0 ; \mathrm{n}=22)$.

CONCLUSÃO: Os resultados foram compatíveis com outras pesquisas que indicaram alta prevalência de SDA em pessoas com HAS e/ou DM. Tais dados reforçam que cuidados com saúde mental devem ser implementados já na atenção primária dessa população.

\section{PALAVRAS-CHAVE: Prevalência; Saúde mental; Hipertensão; Diabetes mellitus}

\section{RESUMEN}

"Prevalencia de síntomas depresivos y/o ansiosos en personas con hipertensión arterial sistémica y/o diabetes mellitus"

CONTEXTO: La Hipertensión Arterial Sistémica (HAS) y la Diabetes Mellitus (DM) se han señalado como condiciones que favorecen el desarrollo de trastornos emocionales.

OBJETIVO: Investigar la prevalencia de síntomas depresivos y/o ansiosos (SDA) en personas con HAS y/o DM registradas en una Estrategia Salud de la Familia del centro-oeste brasileño.

METODOLOGÍA: Se trata de un estudio transversal y cuantitativo. Se investigaron 106 adultos com HAS/DM, a los que se aplicaron dos instrumentos: (1) Mini International Neuropsychiatric Interview para la evaluación de SDA; (2) cuestionario sobre datos socioeconómicos, antropométricos, clínicos y adhesión medicamentosa, para caracterización de la muestra.

RESULTADOS: Los principales hallazgos indicaron la prevalencia de 27,4\% (IC95\% 19,1-36,8, n = 29) de SDA en la muestra total. Además, estos síntomas fueron más prevalentes en los siguientes estratos de esa población: mujeres $(35,4 \%$; IC $95 \% 23,7-48,6 ; n=22)$; las personas con edad avanzada (28,5\%; IC 95\% 14,6 - 46,3, $\mathrm{n}=10)$; las personas sin compañeros $(36,3 \%$; IC95\% 20,4 - 54,8, $\mathrm{n}=12)$; las personas que estudiaron de cero a ocho años (27,8\%; IC95\% 18,3 - 39,0, n = 22).

CONCLUSIÓN: Los resultados fueron compatibles con otras investigaciones que indicaron alta prevalencia de SDA en personas con HAS y/o DM. Tales datos refuerzan que la atención sanitaria mental debe implementarse ya en la atención primaria de esa población.

\section{DESCRIPTORES: Prevalencia; Salud mental; Hipertensión; Diabetes mellitus}

\begin{abstract}
"Prevalence of depressive and/or anxious symptoms in people with systemic arterial hypertension and / or diabetes mellitus"

BACKGROUND: Systemic Arterial Hypertension (SAH) and Diabetes Mellitus (DM) have been identified as conditions that favor the development of emotional disorders.

AIM: To investigate the prevalence of depressive and/or anxious symptoms (SDA) in people with SAH and/or DM enrolled in a Family Health Strategy in the center-west of Brazil.

METHODS: This is a cross-sectional and quantitative study. It was studied 106 adults with SAH / DM to whom two instruments were applied: (1) Mini International Neuropsychiatric Interview, for evaluation of SDA; (2) questionnaire on socioeconomic, anthropometric, clinical data and drug compliance, for characterization of the sample.

RESULTS: The main findings indicated a prevalence of $27.4 \%$ (95\%CI $19.1-36.8, \mathrm{n}=29$ ) of SDA in the total sample. In addition, such symptoms were more prevalent in the following strata of this population: women (35.4\%; 95\%CI 23.7-48.6, $\mathrm{n}=22$ ); people with more advanced age $(28.5 \%$; $95 \%$ CI 14.6 - 46.3; $\mathrm{n}=10$ ); people without partners (36.3\%; 95\%CI 20.4 $54.8, \mathrm{n}=12)$; people who studied from zero to eight years $(27.8 \%$; $95 \% \mathrm{CI}$ $18.3-39.0, \mathrm{n}=22$ ).

CONCLUSION: The results were compatible with other studies that indicated a high prevalence of SDA in people with SAH and/or DM. These data reinforce that mental health care should be implemented already in the primary care of this population.
\end{abstract}

\section{KEYWORDS: Prevalence; Mental health; Hypertension; Diabe- tes Mellitus}

Submetido em 31-03-2018

Aceite em 03-07-2018

1 Psicóloga; Especialista em Arteterapia; Especializanda na Universidade Federal de Mato Grosso, Programa de Residência Multiprofissional em Saúde da Família, Rodovia Rondonópolis-Guiratinga, KM 06 (MT-270), Bairro Sagrada Família, 78735-910, Rondonópolis - Mato Grosso, Brasil, gabrielapsicoarte@gmail.com

2 Psicólogo; Doutor em Psicologia (área de concentração Avaliação Psicológica); Docente na Universidade Federal de Mato Grosso, curso de graduação em Psicologia e programa stricto sensu em Psicologia, 78735-910, Rondonópolis - Mato Grosso, Brasil, rauniroama@gmail.com

3 Enfermeiro; Doutorando em Saúde Pública (área de concentração Epidemiologia) na Universidade Federal de Minas Gerais, Faculdade de Medicina; Professor na Universidade Federal de São João del-Rei, Departamento de Medicina, 36301-182 São João Del Rei - Minas Gerais, Brasil, luis.pauloss@hotmail.com

4 Psicólogo; Doutor em Saúde Pública; Docente na Universidade Federal de Mato Grosso, curso de graduação em Psicologia, Mato Grosso, Brasil, alcindojrosa@gmail.com

Citação: Souza, G. N. P., Alves, R. J. R., Souza, L. P. S., \& Rosa, A. J. (2018). Prevalência de sintomas depressivos e/ou ansiosos em pessoas com hipertensão arterial sistêmica e/ou diabetes mellitus. Revista Portuguesa de Enfermagem de Saúde Mental (20), 43-50. doi: 10.19131/rpesm.0225 


\section{INTRODUÇÃO}

As doenças e agravos crônicos não transmissíveis (DCNT) compõem um cenário complexo e preocupante na área da saúde, pois constituem a principal causa de morbimortalidade no mundo. Dentre as mais frequentes, estão a Hipertensão Arterial Sistêmica (HAS) e o Diabetes Mellitus (DM), que atingem uma em cada três e uma em cada dez pessoas adultas, respectivamente. De acordo com Machado e Campos (2015), suas incidências e prevalências estão associadas à transição demográfica e epidemiológica e, consequentemente, ao envelhecimento populacional. Esses autores afirmam, ainda, que tais condições geram altos custos públicos. Por exemplo, no Brasil, o gasto médio anual com medicamentos no Sistema Único de Saúde (SUS) é R\$ 764.880,00 (aproximadamente $€ 191.220,00$ ).

Além disso, há também custos emocionais para as pessoas com esses quadros, pois estudos apontam que elas apresentam uma maior probabilidade de desenvolverem sintomas depressivos e/ou ansiosos (SDA). Em pesquisa realizada por Helena, Lasagno e Vieira (2010), avaliou-se a prevalência de transtornos mentais não psicóticos (TMNP), dentre eles SDA, em pessoas com HAS e/ou DM oriundas de Unidades de Saúde da Família de Blumenau, Santa Catarina, Brasil. Os selecionados $(\mathrm{N}=710)$ foram submetidos a um questionário domiciliar com questões relativas às características socioeconômicas, pessoais e de medicamentos, e ao SelfReport Questionnaire-20 (SRQ-20). As variáveis estudadas foram submetidas à análise uni e multivariada. A prevalência desses transtornos foi de $39,4 \%$ e os fatores de maior associação a eles foram: ser do sexo feminino $(\mathrm{RP}=1,50 ; \mathrm{IC} 95 \% 1,12-2,01 ; \mathrm{p}<0,01)$, fazer uso de psicotrópicos ( $\mathrm{RP}=1,74$; IC95\% 1,34-2,26; $\mathrm{p}<0,01)$, ter ficado hospitalizado e/ou alta frequência de comparecimento ao pronto-socorro no último ano $(\mathrm{RP}=1,30$; IC95\% 1,02-1,66; $\mathrm{p}=0,03)$ e ser da classe econômica de consumo "E" ( $R P=2,62$; IC95\% 1,09 - 6,27; p = 0,03), de acordo com a classificação da Associação Brasileira de Empresas de Pesquisa (ABEP).

Por sua vez, Oliveira e Sales (2005) buscaram verificar a ocorrência de SDA em indivíduos $(\mathrm{N}=68)$ com HAS e/ ou DM atendidos por uma Equipe de Saúde da Família, bem como a adesão ao tratamento e apoio familiar. As autoras aplicaram algumas entrevistas sobre aspectos pessoais, uma escala que visava aferir SDA e realizaram a medição de glicemia e da pressão arterial em todos os participantes.
Os resultados apontaram que $72 \%$ apresentaram SDA, e que, além disso, 54,4\% não praticam atividades físicas, $55,9 \%$ apresentaram glicemia e alterações nos níveis pressóricos. As autoras inferiram possível influência da SDA na adesão ao tratamento e qualidade de vida dessa população. Além disso, apontou a importância dos grupos de educação em saúde como forma de suporte.

Amaral et al. (2007) realizaram uma investigação mais específica, na qual buscaram identificar a prevalência de transtorno depressivo maior em 285 pessoas somente com HAS. Aplicaram o Inventário de Depressão de Beck (BDI) e a "Entrevista Estruturada para o Manual de Diagnóstico e Estatística das Perturbações Mentais - Transtornos do Eixo I" para o rastreamento desses sintomas, tendo encontrado prevalência de $20 \%$.

Por outro lado, Braz et al. (2012) investigaram um grupo de pessoas somente com DM $(\mathrm{N}=145)$, buscando averiguar a associação entre sintomas depressivos, variáveis sociodemográficas e clínicas e adesão ao tratamento medicamentoso. Aplicaram-se o BDI e a Medida de Adesão aos Tratamentos (MAT). O BDI indicou a prevalência de sintomas depressivos igual a 33,1\%. Interessantemente, a prática de atividade física se associou com uma melhor avaliação no BDI $(p=0,05)$, mas não foi observada correlação clinicamente importante entre o BDI e o MAT.

Lustman e Clouse (2005) também buscaram investigar evidências da comorbidade entre diabetes e o risco de depressão. Realizaram um estudo de revisão da literatura nas bases de dados PubMed, PsycINFO e MEDLINE, com os seguintes descritores: diabetes, depressão, controle metabólico, hiperglicemia, hipoglicemia; com delimitação de tempo de 1980 e 2002.

De modo geral, os estudos encontrados evidenciaram que a depressão, quando presente em pessoas com diabetes, favorece diminuição no controle metabólico, baixa adesão à medicação e aos regimes dietéticos, redução da qualidade de vida e aumento dos gastos com cuidados de saúde.

Vericaram, também, que um controle metabólico fraco pode exacerbar a depressão e diminuir a resposta aos antidepressivos.

A psicoterapia e a farmacoterapia foram apontados como tratamentos que devem se fazer presentes nessa população.

Para os autores, os esforços aprimorados para um bom controle glicêmico podem contribuir para melhorias no humor e nas percepções de bem-estar. 
No entanto, apesar desses dados indicarem a presença dessas sintomatologias emocionais de modo contundente nos casos de HAS e DM, no contexto da Atenção Primária à Saúde (APS) do Brasil ainda são incipientes as propostas de avaliação e de intervenção que consideram os processos psicológicos como parte da multifatorialidade envolvida em seus cuidados, bem como em de outras condições.

A atual política de saúde mental brasileira alimentouse de experiências exitosas de países europeus na substituição de um modelo de saúde mental baseado em hospitalização psiquiátrica por um modelo de serviços comunitários com forte inserção territorial.

O Ministério da Saúde do Brasil (2013) divulgou, no caderno 34, diretrizes e ferramentas para esse modelo de cuidado em saúde mental na APS, compreendendo esse nível de atenção como um serviço estratégico que pode ser realizado por todos que compõem a equipe multiprofissional.

Abrange desde estratégias de acolhida e momentos de reflexão, tendo como legítima a habilidade de empatia como uma intervenção em saúde mental que deve ser comum a todos os atuantes na área. Porém, um dos passos iniciais é reconhecer sintomatologias diversas, bem como compreender que algumas podem se fazer mais prevalentes, prática essa ainda incomum no cotidiano dos atendimentos realizados.

O Apoio Matricial oferecido pelas equipes de Núcleos de Apoio à Saúde da Família (NASF) embasam a integralidade do cuidado e a responsabilização compartilhada dos casos entre equipes de Estratégia Saúde da Família (ESF), nesse nível da atenção. O que unifica o objetivo dessas equipes deve ser o entendimento do território e a relação de vínculo dos profissionais de saúde com os usuários.

Essas políticas são aplicadas em todo território brasileiro, porém, há certa carência quando pensado ainda o centro-oeste do país. Essa carência envolve desde a realização de pesquisas de prevalência, como as supracitadas no início do presente texto, bem como o funcionamento prático dos atendimentos dos aspectos de saúde mental também já citados.

Deste modo, a fim de reforçar o cuidado dos aspectos já na Atenção Primária à Saúde, principalmente relativos ao centro-oeste brasileiro, o presente estudo se propôs a investigar a prevalência de SDA em pessoas com HAS e/ou DM cadastradas em uma ESF presente nessa localização.

\section{MÉTODOS}

Trata-se de um estudo transversal e quantitativo, realizado com 106 participantes com HAS e/ou DM provindos de uma ESF do distrito sul do município de Rondonópolis, Mato Grosso, Brasil. O estudo foi aprovado pelo Comitê de Ética em Pesquisa da Universidade Federal de Mato Grosso (CAAE 45225315.0.0000.5541), tendo sido realizado entre dezembro de 2016 e julho de 2017.

O levantamento dos usuários com HAS e/ou DM foi realizado por meio da análise prévia dos prontuários, e, posteriormente, visita domiciliária. Foram considerados os seguintes critérios de inclusão: ser maior de 20 anos; residir na área de abrangência da ESF investigada; e, para as mulheres, também se adotou a condição de não ser gestante. Os de exclusão foram: algum comprometimento que não permitisse condições de resposta aos instrumentos (como deficiências e/ou transtornos psiquiátricos) e aqueles participantes que não fossem encontrados em seus domicílios em até três tentativas de contato. Inicialmente, foram incluídos no estudo 174 participantes, contudo, após exclusões, a amostra final foi composta por 106 .

Durante a visita domiciliária, foram aplicados dois instrumentos de autorrelato, que somente eram respondidos após os participantes lerem e assinarem o Termo de Consentimento Livre Esclarecido. Um dos instrumentos foi a Versão Brasileira 5.0.0 da entrevista diagnóstica padronizada breve Mini International Neuropsychiatric Interview (MINI) (Lecrubier et al., 2000), utilizada para caracterização dos sintomas de ansiedade e depressão. É organizada por 19 módulos que exploram 17 transtornos do Eixo I do DSM-IV, o risco de suicídio e o transtorno da personalidade anti-social. Identifica sinais diagnósticos positivos para os principais transtornos psicóticos e do humor.

Para a presente pesquisa, foram utilizadas somente as seções $\mathrm{A}, \mathrm{B}$ e $\mathrm{O}$ do instrumento, que possibilitam a identificação de sintomas característicos aos quadros de: episódio depressivo maior atual; episódio depressivo maior recorrente; transtorno distímico atual; e transtorno de ansiedade generalizada atual.

Os participantes foram classificados com "SDA" quando apresentaram resultado positivo para pelo menos um desses quadros.

Utilizou-se, ainda, um questionário para levantar os dados socioeconômicos, antropométricos, clínicos e de adesão medicamentosa. 
As prevalências de DAS aferidas pelo MINI e seus respectivos intervalos de confiança foram analisados de acordo com variáveis extraídas do questionário aplicado. Algumas dessas variáveis foram organizadas em estratos populacionais a fim de se extrair conclusões mais abrangentes e generalizáveis para a amostra estudada, que não foi tão ampla. Primeiramente, organizou-se a idade, dividindo-a em dois grandes grupos para análise dessas sintomatologias: adultos (de 20 a 59 anos) e idosos (60 anos ou mais). Por meio dessas classificações, buscou-se identificar os impactos de senilidade sobre os sintomas.

A escolaridade foi categorizada em duas faixas: (a) zero a oito anos de estudo; (b) nove ou mais anos de estudo. Aferiu-se, desse modo, que as pessoas pertencentes à primeira faixa teriam tido acesso ao denominado Ensino Fundamental no Brasil, podendo ter um nível de escolaridade de analfabeto até de domínio satisfatório da leitura, escrita e do cálculo. Já na segunda, aferiu-se que as pessoas poderiam ter tido acesso desde ao denominado "Ensino Médio" até a pós-graduação, o que indicaria, provavelmente, capacidades mais elaboradas de leitura, escrita e raciocínio lógico e de diversos outros conhecimentos sociais, bem como um acesso a profissões mais estáveis e mais bem remuneradas. Buscouse identificar os impactos da escolarização, de acordo com classificações adotadas no Brasil.

As rendas foram classificadas por meio dos salários mínimos: até meio, de meio a dois, de dois a cinco e de cinco a dez. As classes econômicas foram compatíveis a tais salários, sendo a classe "A-B" compatível a uma renda de mais de cinco salários mínimos (acima de $\mathrm{R} \$$ 4.780, aproximadamente, acima de € 1.195) e "C-D" de menos de cinco salários mínimos (Associação Brasileira de Empresas de Pesquisa, 2016). Essas classificações favoreceriam verificar a análise das sintomatologias considerando o impacto econômico, também considerando classificações brasileiras.

Foram consideradas variáveis menos possíveis de organização em estratos, tais como: raça ou cor da pele autodeclarada (branca; amarela; parda; indígena; preta) (Instituto Brasileiro de Geografia e Estatística (IBGE), 2013), sexo (masculino; feminino), estado civil (com companheiro(a); sem companheiro(a)), trabalho (sim; não), moradia (própria; alugada), doença (HAS; DM; HAS e DM) e hábitos de vida (sedentarismo; tabagismo; uso diário de álcool; excesso de peso; adesão medicamentosa (baixa/mediana; elevada) (Morisky, Green \& Levine, 1986).
O banco de dados foi digitado no Microsoft Office Excel $^{\circledast}$ 2007. A verificação e a tabulação do número de participantes, porcentagens e intervalos de confiança 95\% (IC95\%) foram realizadas utilizando o programa Epi Info ${ }^{\oplus}$, versão 3.5.4.

\section{RESULTADOS}

A prevalência de SDA na amostra geral $(\mathrm{N}=106)$ foi de $27,4 \%$ (IC95\% = 19,1 - 36,8; $\mathrm{n}=29$ ). Maior prevalência foi encontrada em mulheres, nas pessoas com idade mais avançada, nos que não possuíam companheiros e nos que estudaram de zero a oito anos. Os participantes que se autodeclararam pertencer a raça/cor branca apresentaram menor prevalência desses sintomas, seguidamente pelas pessoas de cor parda, preta e indígena (Tabela 1).

Tabela 1 - Prevalência de Sintomas Depressivos e/ou Ansiosos de Acordo com as Características Sociodemográficas dos Participantes $(\mathrm{N}=106)$. Rondonópolis, Mato Grosso, Brasil, 2017.

\begin{tabular}{|c|c|c|}
\hline Variável & n $(\% ; \mathrm{IC} 95 \%)^{*}$ & $\begin{array}{l}\text { Prevalência de sintomas } \\
\text { depressivos e/ou ansiosos } \\
\text { n }(\% \text {; IC } 95 \%)^{\star *}\end{array}$ \\
\hline \multicolumn{3}{|l|}{ Sexo } \\
\hline Masculino & $44(41,5 ; 32,0-51,4)$ & $7(15,9 ; 6,6-30,0)$ \\
\hline Feminino & $62(58,4 ; 48,5-67,9)$ & $22(35,4 ; 23,7-48,6)$ \\
\hline \multicolumn{3}{|l|}{ Idade (anos) } \\
\hline $20-59$ & $71(66,9 ; 57,1-75,8)$ & $19(26,7 ; 16,9-38,5)$ \\
\hline$\geq 60$ & $35(33,0 ; 24,1-42,8)$ & $10(28,5 ; 14,6-46,3)$ \\
\hline \multicolumn{3}{|l|}{ Estado civil } \\
\hline $\begin{array}{l}\text { Com com- } \\
\text { panheiro (a) }\end{array}$ & $73(68,8 ; 59,1-77,5)$ & $17(23,2 ; 14,1-34,6)$ \\
\hline $\begin{array}{l}\text { Sem com- } \\
\text { panheiro (a) }\end{array}$ & $33(31,1 ; 22,4-40,8)$ & $12(36,3 ; 20,4-54,8)$ \\
\hline \multicolumn{3}{|l|}{ Escolaridade } \\
\hline 0 a 8 anos & $79(74,5 ; 65,1-82,4)$ & $22(27,8 ; 18,3-39,0)$ \\
\hline+ de 9 anos & $27(25,4 ; 17,5-34,8)$ & $7(25,9 ; 11,1-46,2)$ \\
\hline \multicolumn{3}{|l|}{ Raça/Cor } \\
\hline Amarela & $1(0,9 ; 0,02-5,1)$ & 0 \\
\hline Branca & $16(15,0 ; 8,8-23,3)$ & $2(12,5 ; 1,55-38,3)$ \\
\hline Indígena & $2(1,8 ; 0,2-6,6)$ & $1(50 ; 1,26-98,7)$ \\
\hline Parda & $61(57,5 ; 47,5-67,0)$ & $16(26,2 ; 15,8-39,0)$ \\
\hline Preta & $26(24,5 ; 16,6-33,8)$ & $10(38,4 ; 20,2-59,4)$ \\
\hline
\end{tabular}

Legenda: ${ }^{*}$ Número de participantes, porcentagem e intervalo de confiança na população investigada $(\mathrm{N}=106) ;{ }^{* *}$ Número de participantes, porcentagem e intervalo de confiança de sintomas depressivos e ansiosos presentes em cada estrato populacional investigado. 
Na Tabela 2, é possível observar que características de SDA tiveram maior prevalência nos casos comórbidos de HAS e DM, seguidos por aqueles que somente tinham HAS e, por fim, DM. Em relação aos hábitos de vida, maior prevalência foi encontrada em usuários que faziam uso de álcool diariamente, seguido por aqueles com excesso de peso, sedentários e tabagistas. Foi verificada, também, que a sintomatologia esteve mais presente naqueles cuja adesão medicamentosa estava em nível baixo/mediano (Tabela 2).

Tabela 2 - Prevalência de Sintomas Depressivos e/ou Ansiosos de Acordo com as Características de Saúde dos Participantes ( $\mathrm{N}=$ 106). Rondonópolis, Mato Grosso, Brasil, 2017.

\begin{tabular}{|c|c|c|}
\hline Variável & n $(\% ;$ IC95\%)* & $\begin{array}{l}\text { Prevalência de sin- } \\
\text { tomas depressivos } \\
\text { e/ou ansiosos } \\
\text { n }(\% ; \mathrm{IC} 95 \%)^{* *}\end{array}$ \\
\hline \multicolumn{3}{|l|}{ Doença } \\
\hline Hipertensão & $76(71,7 ; 62,1-80,0)$ & $21(27,6 ; 17,9-39,0)$ \\
\hline Diabetes Mellitus & $9(8,4 ; 3,9-15,5)$ & $2(22,2 ; 2,8-60,0)$ \\
\hline $\begin{array}{l}\text { Hipertensão e } \\
\text { Diabetes }\end{array}$ & $21(19,8 ; 12,7-28,6)$ & $6(28,5 ; 11,2-52,1)$ \\
\hline \multicolumn{3}{|l|}{ Hábitos de vida } \\
\hline Sedentarismos & $58(54,7 ; 44,7-64,4)$ & $17(29,3 ; 18,0-42,7)$ \\
\hline Tabagismo & $22(20,7 ; 13,4-29,7)$ & $4(18,1 ; 5,1-40,2)$ \\
\hline $\begin{array}{l}\text { Uso de álcool } \\
\text { diário }\end{array}$ & $2(1,8 ; 0,2-6,6)$ & $2(100 ; 15,8-100)$ \\
\hline Excesso de peso & $52(49,0 ; 39,2-58,9)$ & $17(32,6 ; 20,3-47,1)$ \\
\hline \multicolumn{3}{|c|}{ Adesão medicamentosa } \\
\hline Baixa/Mediana & $37(34,9 ; 25,9-44,7)$ & $11(29,7 ; 15,8-46,9)$ \\
\hline Elevada & $69(65,0 ; 55,2-74,1)$ & $18(26,0 ; 16,2-38,0)$ \\
\hline
\end{tabular}

Legenda: ${ }^{*}$ Número de participantes, porcentagem e intervalo de confiança na população investigada $(n=106)$; ${ }^{* *}$ Número de participantes, porcentagem e intervalo de confiança de sintomas depressivos e ansiosos presentes em cada estrato populacional investigado.

$\mathrm{Na}$ Tabela 3, pode-se observar que os DAS foram mais prevalentes naqueles cuja renda foi de até meio salário mínimo, seguidos por aqueles com dois a cinco salários e, por fim, daqueles com meio a dois salários. Foram mais prevalentes naqueles que pertenciam às classes econômicas $\mathrm{C}$ e $\mathrm{D}$, quando comparados àqueles das classes A e B. Maior prevalência foi vista nos que disseram não trabalhar, bem como naqueles com moradia alugada (Tabela3).
Tabela 3 - Prevalência de Sintomas Depressivos e/ou Ansiosos de Acordo com as Características Econômicas dos Participantes ( $\mathrm{N}=106)$. Rondonópolis, Mato Grosso, Brasil, 2017.

\begin{tabular}{|c|c|c|}
\hline Variável & $\begin{array}{l}\text { n }(\% ; \\
\text { IC95\%)* }\end{array}$ & $\begin{array}{l}\text { Prevalência de } \\
\text { sintomas depressi- } \\
\text { vos e/ou ansiosos } \\
\text { n }(\% \text {; IC } 95 \%)^{\star \star}\end{array}$ \\
\hline \multicolumn{3}{|l|}{ Renda } \\
\hline $\begin{array}{l}\text { Até } 1 / 2 \text { salário mínimo } \\
(\mathrm{R} \$ 468,50)\end{array}$ & $\begin{array}{l}76(71,7 ; \\
62,1-80,0)\end{array}$ & $\begin{array}{l}21(27,6 ; 17,9- \\
39,0)\end{array}$ \\
\hline $\begin{array}{l}\text { De } 1 / 2 \text { a } 2 \text { salários mínimos } \\
(\mathrm{R} \$ 468,50-1.874,00)\end{array}$ & $\begin{array}{l}68(64,1 ; \\
54,2-73,2)\end{array}$ & $\begin{array}{l}15(22,0 ; 12,9- \\
33,7)\end{array}$ \\
\hline $\begin{array}{l}\text { De } 2 \text { a } 5 \text { salários mínimos } \\
(\mathrm{R} \$ 1.874,00-4.685,00)\end{array}$ & $\begin{array}{l}27(25,4 ; \\
17,5-34,8)\end{array}$ & $8(29,6 ; 13,7-50,1)$ \\
\hline $\begin{array}{l}\text { De } 5 \text { a } 10 \text { salários mínimos } \\
(\mathrm{R} \$ 4,685,00-9.370,00)\end{array}$ & $\begin{array}{l}1(0,9 ; 0,02 \\
-5,1)\end{array}$ & 0 \\
\hline \multicolumn{3}{|l|}{ Classe econômica } \\
\hline A e B (+5 salários mínimos) & $\begin{array}{l}1(0,9 ; 0,94 \\
0,02-5,1)\end{array}$ & 0 \\
\hline C e D (-5 salários mínimos) & $\begin{array}{l}105(99,0 ; \\
94,8-99,9)\end{array}$ & $\begin{array}{l}29(27,6 ; 19,3- \\
37,2)\end{array}$ \\
\hline \multicolumn{3}{|l|}{ Trabalho } \\
\hline Sim & $\begin{array}{l}45(42,4 ; \\
32,9-52,4)\end{array}$ & $7(15,5 ; 6,4-29,4)$ \\
\hline Não & $\begin{array}{l}61(57,5 \\
47,5-67,0)\end{array}$ & $\begin{array}{l}22(36,0 ; 24,1- \\
49,3)\end{array}$ \\
\hline \multicolumn{3}{|l|}{ Moradia } \\
\hline Própria & $\begin{array}{l}99(93,4 ; \\
86,8-97,3)\end{array}$ & $\begin{array}{l}27(27,2 ; 18,8- \\
37,1)\end{array}$ \\
\hline Alugada & $\begin{array}{l}7(6,6 ; 2,7- \\
13,1)\end{array}$ & $2(28,5 ; 3,6-70,9)$ \\
\hline
\end{tabular}

Legenda: ${ }^{\star}$ Número de participantes, porcentagem e intervalo de confiança na população investigada $(\mathrm{n}=106) ;{ }^{* *}$ Número de participantes, porcentagem e intervalo de confiança de sintomas depressivos e ansiosos presentes em cada estrato populacional investigado.

\section{DISCUSSÃO}

Nesse estudo, a prevalência encontrada de SDA em pessoas com HAS e DM foi de $27,4 \%$, e, apesar de baixa quando comparada a investigações anteriores com a mesma população (Helena, Lasagno e Vieira, 2010; Oliveira e Sales, 2005), é considerada alta quando comparada aos dados nacionais referente à população geral, que apontam o percentual de 7,6\% para depressão e 6,4\% para transtornos de ansiedade (IBGE, 2014). Tal dado reforça a necessidade de avaliações periódicas no que diz respeito às sintomatologias em pacientes com HAS e DM na APS, uma vez que um diagnóstico precoce possibilita uma terapêutica mais eficaz e menos custosa. 
Verificou-se maior prevalência do desfecho na população feminina, resultado parecido com aqueles encontrados por outras pesquisas (Helena, Lasagno e Vieira, 2010; Oliveira e Sales, 2005). Alguns estudos apontam que a maior prevalência de depressão na população geral de mulheres, quando comparada à dos homens, pode estar associada às questões ligadas ao gênero. A sobrecarga das mulheres, relacionada à dupla jornada de trabalho e até mesmo ao estado civil, como de ser solteira/viúva ou divorciada, podem contribuir para tal predisposição (Almeida et al., 2004). Quando analisado o estado civil, a amostra deste estudo foi composta, em sua maioria, por mulheres $(58,4 \%)$, obtendo maior prevalência da DAS naquelas que referiram estar "Sem companheiro(a)". Possivelmente, o desvalor cultural e a falta de um apoio cotidiano mais próximo justifiquem este resultado.

Maior prevalência de SDA $(28,5 \%)$ foi encontrada nos participantes com faixa etária entre 60 anos ou mais, corroborando com os dados da Pesquisa Nacional de Saúde (PNS) a qual aponta que, na população geral brasileira, a faixa etária com maior proporção de depressão é a de 60 a 64 anos de idade (11\%) (IBGE, 2014). Em outra pesquisa brasileira, foi verificado que as prevalências de transtornos psiquiátricos menores, como as investigadas neste estudo, apresentaram-se mais frequentes a partir dos 40 anos de idade, com tendência a aumentar ano após ano, também na população geral (Costa, et al., 2002). Em estudo específico de pessoas com HAS e DM, houve maior tendência de desenvolver depressão entre os idosos (Oliveira e Sales, 2005).

Do ponto de vista vivencial, o processo de envelhecimento se dá com experiências de perdas continuadas, seja com a falta de status ocupacional, o valor econômico, agravamento de condições clínicas e até mesmo declínio progressivo de humor causado pelas mudanças do sistema endócrino, neurológico e fisiológico, próprias da idade.

Por sua vez, a vivência desse processo biopsicossocial pode gerar, muitas vezes, quadros de sofrimento psíquico (Guimarães e Podkameni, 2012). Aventou-se que esses processos façam parte, até mesmo em maior grau, na população aqui investigada.

Por sua vez, nos resultados observados quanto à variável raça/cor, obteve-se que a prevalência de SDA foi maior na população autodeclarada indígena (50\%), seguida da preta $(38,4 \%)$, parda $(26,2 \%)$ e branca $(12,5 \%)$. Alguns estudos apontam que desigualdades étnico-raciais são agravantes para o sofrimento em saúde mental.
O racismo e a negação de direitos que dele decorre são recorrentes no processo de construção de mal-estar ou adoecimento psíquico (Guimarães e Podkameni, 2012). Em relação à população indígena na zuna urbana, alguns fatores que tendem a fomentar transtornos psicológicos seria a inferiorização social, imposição de adaptação a outra cultura, a barreira linguística e o julgamento social do índio na cidade (Rodrigues, 2014). Na população aqui investigada, supõe-se que tal impacto também se fez presente.

Pôde-se verificar maiores sintomatologias de SDA nos estratos de classe econômica baixa (C e D), menor escolaridade (0 a 8 anos) e de não trabalho. Ludemir e Melo (2002) apontam que a baixa escolaridade, baixa renda e exclusão do mercado formal de trabalho estão relacionadas e são situações estressoras contribuintes para a origem de transtornos mentais. Além disso, especificamente o grau de escolaridade reflete condições socioeconômicas e influencia comportamentos e escolhas futuras, com sofrimento psíquico mais comum a pessoas com posições mais desfavoráveis de acesso escolar. Nos participantes desta pesquisa, é possível que tais posições tenham tido esse efeito.

Verificou-se, também, que nos participantes com HAS, isoladamente, foi encontrada maior sintomatologia de SDA do que nos com DM. Não foram encontrados estudos na literatura que compararam as duas condições quanto a sintomatologia. Porém, Saboya (2009) evidenciou que as chances de um indivíduo com HAS apresentar DAS são de aproximadamente três a quatro vezes maiores que as de um indivíduo normotenso. Prevalência semelhante seria esperada em pessoas com DM, de acordo com Moussavi et al. (2007), que analisaram dados de indivíduos adultos de 60 países. Pode ser que o fato de a amostra ser composta por um número bem maior de participantes com HAS $(n=76)$ quando comparados ao somente com DM $(n=9)$ tenha influenciado tal prevalência no estudo de Moussavi et al. (2007). Verificou-se maior prevalência de SDA nos quadros comórbidos de HAS e DM.

$\mathrm{Na}$ literatura nacional, não foram encontrados estudos que comparassem grupos comórbidos com não-comórbidos dessas condições. Mas, seguindo um princípio lógico, tal dado poderia ser explicado pelo fato das duas condições estarem presentes concomitantemente, e, dessa forma, favorecerem ainda mais a prevalência dos SDA.

A prevalência de DAS foi maior naqueles que apresentaram baixa/média adesão medicamentosa. 
Infelizmente, pode-se verificar que a adesão à farmacoterapia pode ser prejudicada pela presença destes sintomas, o que é compatível com achados anteriores realizados tanto com a população geral como com pessoas com DM (Braz et al., 2012).

Por sua vez, a prevalência de SDA esteve mais presente em usuários que referiram consumir álcool diariamente. Esses dados vão ao encontro de estudos que apontam que na população geral esse consumo está atrelado frequentemente a transtornos mentais não psicóticos, como os aqui estudados (Costa et al.,2002; Moreira et al.,1996). O álcool possui efeito ansiolítico/relaxante sobre o comportamento humano, o que favorece em muito o alto consumo em populações de risco (Moreira et al.,1996), como a aqui estudada.

É importante reforçar que uma das limitações do presente estudo é a distribuição dos dados amostrais e estratificados. Por exemplo, foram investigados somente dois indígenas e dois usuários que faziam uso de álcool diariamente. Tais dados, por serem muito pequenos, podem ter levado a prevalências não tão generalizáveis para a população estudada, apesar de terem sido compatíveis com a literatura. Sugere-se que em estudos futuros, possam-se aumentar todos os estratos com baixo número de participantes. Tal controle favoreceria uma análise mais detalhada, como, por exemplo, de faixas etárias e tempos de escolaridade menos amplos e mais diversificados.

Além disso, a amostra total foi obtida por conveniência, não sendo estabelecido um cálculo amostral prévio para que se pudesse atingir uma amostra ideal (ou próxima ao ideal) para ser pesquisada no município de Rondonópolis, local foco da investigação. Sugere-se que, futuramente, esse controle e ampliação da pesquisa sejam realizados.

Uma outra e última limitação observada foi em relação às próprias sintomatologias investigadas. Ressalta-se que aqui não foi investigado se elas já se caracterizariam como transtornos depressivos e ansiosos, somente foram identificados sinais de suas presenças pelo instrumento MINI. Sugere-se que, em pesquisas futuras, seja identificada a presença desses transtornos propriamente ditos. Dessa forma, aconselha-se cuidado para a generalização dos dados aqui encontrados em âmbito psiquiátrico.

\section{CONCLUSÃO}

O presente estudo cumpriu seu objetivo de investigar a prevalência de SDA em pessoas com HAS e/ou DM cadastradas em uma ESF do centro-oeste brasileiro.
A hipótese de que a prevalência dessa sintomatologia seria alta nessa localização foi confirmada, justificando ainda mais este tipo de serviço na região e em outras localidades do país. Permitiu reforçar a relevância do trabalho dos profissionais da saúde em identificar aspectos emocionais que possam influenciar o tratamento das DCNT. Os SDA foram mais prevalentes na população estudada com HAS e DM do que na população geral, de acordo com a literatura. Ressalta-se que cuidados sobre a saúde mental dessa população devem ser melhor trabalhados e/ou estabelecidos no contexto da ESF.

\section{IMPLICAÇÕES PARA A PRÁTICA CLÍNICA}

Esse estudo permitiu verificar que o uso de instrumentos que auxiliem no rastreamento de SDA é de grande significância na atenção primária a saúde. A entrevista diagnóstica padronizada breve MINI se mostrou como uma ferramenta de grande potencial nesse nível de assistência, pois permitiu a identificação de SDA de modo prático, simples e de fácil acesso e compreensão por todos os membros da equipe.

Os dados apontaram, também, para necessidade de serem consideradas as multifatorialidades das DCNT no âmbito da atenção primária à saúde. A investigação por estratos permitiu identificar que para uma assistência mais efetiva e singular, é necessário que os profissionais abranjam em suas práticas os aspectos biopsicossociais dos usuários, e que projetem intervenções que abranjam todas essas esferas numa dinâmica de trabalho interdisciplinar. Estratégias como promoção e prevenção de saúde, ações intersetoriais, capacitação em saúde mental, inclusão de equipes de NASF nas ESF's, discussão de casos, interconsultas e construção conjunta de projetos terapêuticos são modos de se trabalhar a saúde integral desta população.

\section{REFERÊNCIAS BIBLIOGRÁFICAS}

Almeida, F. N., Lessa I., Magalhães, G., Araújo, M.J., Aquino, E., James, A. S., \& Kawachi E. U. (2004). Social inequality and depressive disorders in Bahia, Brazil: interactions of gender, ethnicity and social class. Social Science \& Medicine, 59 (7), 1339-1353. Doi: 10.1016/j. socscimed.2003.11.037

Amaral, G. F., Jardim P. C. B. V., Brasil, M. A. A., Souza, A. L. L., Freitas, H. F., Melo, A. F. B., e Ribeiro, C. N. (2007). Prevalência de transtorno depressivo maior em centro de referência no tratamento de hipertensão arterial. Revista de Psiquiatria do Rio Grande do Sul, 29 (2), 161-168. Doi: 10.1590/S0101-8108200700020000 
Associação Brasileira de Empresas de Pesquisa. (2016). Critério de classificação econômica Brasil: Critério Brasil 2015 e atualização da distribuição de classes para 2016. Disponível em: http://www.abep.org/criteriobrasil

Brasil, Ministério da Saúde, Secretaria de Atenção à Saúde (2013). Cadernos de atenção básica: Saúde Mental. Brasília: Ministério da Saúde.

Braz, J. M., Silva, M. R., Gois, C. F. L., Braz, T. M., Santos, V., e Silva, L. A. S. M.(2012). Sintomas depressivos e adesão ao tratamento entre pessoas com diabetes mellitus tipo 2. Revista Rede de Enfermagem do Nordeste, 13 (5), 1092-99.

Costa, J. S. D., Menezes, A. M. B., Olinto, M. T. A., Gigante, D. P., Macedo, S., Britto, M. A. P., e Fuchs, S. C. (2002). Prevalência de distúrbios psiquiátricos menores na cidade de Pelotas, RS. Revista Brasileira de Epidemiologia, 5 (2), 164-73. Doi: 10.1590/S1415790X2002000200004

Guimarães, M. A. C., e Podkameni, A. B. (2012). Racismo: mal-estar psíquico. In: Batista, L. E., Werneck, J., e Lopes, F. Saúde da População Negra. 2aEd. (pp. 224239). Brasília: Associação Brasileira de Pesquisadores Negros.

Helena, E. T. S., Lasagno, B. G. S., e Vieira, R. (2010). Prevalência de transtornos mentais não-psicóticos e fatores associados em pessoas com hipertensão arterial sistêmica e/ou diabetes mellitus em Unidades de Saúde da Família em Blumenau, Santa Catarina. Revista Brasileira de Medicina da Família e Comunidade, 17 (5), 42-47.

Instituto Brasileiro de Geografia e Estatística. (2014). Pesquisa Nacional de Saúde 2013: percepção do estado de saúde, estilos de vida e doenças crônicas - Brasil, Grandes Regiões e Unidades da Federação. Rio de Janeiro: IBGE.

Instituto Brasileiro de Geografia e Estatística. (2013). Características Étnico-Raciais da População: classificações e identidades. Rio de Janeiro: IBGE.

Lecrubier, Y., Weiller, E., Hergueta, T., Amorim, P., Bonora, L.I., e Lépine, J.P. (2000). The Mini International Neuropsychiatric Interview (MINI): Brazilian version 5.0.0.
Ludermir, A. B., e Melo, F. D. A. (2002). Condições de vida e estrutura ocupacional associadas a transtornos mentais comuns. Revista de Saúde Pública, 36 (2), 21321.

Lustman, P. J., \& Clouse, R. E. (2005). Depression in diabetic patients: the relationship between mood and glycemic control. Diabetes Journal and its Complications, 19 (2), 113-22. Doi: 10.1016 / j.jdiacomp.2004.01.002

Machado, L. E., e Campos, R. (2015). O impacto da diabetes melito e da hipertensão arterial para a saúde pública. Saúde e meio ambiente: revista interdisciplinar, 3 (2), 53-61. Disponível em: http://www.periodicos. unc.br/index.php/sma/article/view/627/497

Moreira, L. B., Fuchs, F. D., Moraes, R. S., Bredemeier, M., Cardozo, S., Fuchs, S. C., \& Victora, C. G. (1996). Alcoholic beverage consumption and associated factors in Porto Alegre, a southern Brazilian city: a populationbased survey. Journal of Studies on Alcohol, 57 (3), 253-259.

Morisky, D. E., Green, L. W., \& Levine, D. M. (1986). Concurrent and predictive validity of a self-reported measure of medication adherence. Medical Care, 24 (1), 67-74. Doi: 10.1097/00005650-198601000-00007

Moussavi, S., Chatterji, S., Verdes, E., Tandon, A., Patel, V., \& Ustun, B. (2007). Depression, chronic diseases, and decrements in health: results from the World Health Surveys. The Lancet, 370 (9590), 851-858. Doi: 10.1016/S0140-6736(07)61415-9

Oliveira, A. R., e Sales, C. A. C. (2005). Ansiedade e Depressão em Clientes com Hipertensão e Diabetes Atendidos por uma Equipe de Saúde da Família. Saúde Coletiva, 2 (6), 53-56. Disponível em: http://www.redalyc. org/articulo.oa? id=84220795004

Rodrigues, R. A. (2014). Sofrimento mental de indígenas na Amazônia. Revista Eletrônica Mutações, 8 (5). Disponível em: http://www.periodicos.ufam.edu.br/ relem/article/view/785/pdf

Saboya, P. M. H. P. (2009). Estudo sobre associação entre hipertensão arterial, ansiedade e sintomas depressivos. (Dissertação de Mestrado). Pontifícia Universidade Católica do Rio Grande Do Sul, Brasil. 